\title{
Formation of a condensed state with macroscopic number of phonons in ultracold Bose gases
}

\author{
Yu. Kagan and L.A. Manakova* \\ RRC "Kurchatov Institute", 123182 Kurchatov Sq.,1, Moscow, Russia
}

\begin{abstract}
A mechanism for the formation of a new type of stationary state with macroscopical number of phonons in condensed atomic gases is proposed. This mechanism is based on generating longitudinal phonons as a result of parametric resonance caused by a permanent modulation of the transverse trap frequency in an elongated trap. The phonon-phonon interaction predetermines the self-consistent evolution which is completed with macroscopic population of one from all levels within the energy interval of parametric amplification. This level proves to be shifted to the edge of this interval. All other levels end the evolution with zero population.
\end{abstract}

PACS numbers: 03.75.Kk, 03.75.Nt, 67.40.Db

\section{INTRODUCTION}

At zero temperature the formation of the stationary state in an atomic condensate with involving of macroscopical number of phonons certainly requires thermodynamically non-equilibrium conditions.

In the present paper an idea is proposed for realizing these conditions for longitudinal sound phonons in elongated Bose-condensed ultracold gases. The idea is based on generating phonons as a result of the parametric resonance induced by a permanent modulation of the transverse trap frequency $\omega_{\perp}$. In last years, it has become clear that the parametric resonance plays an important role in a number of phenomena in ultracold gases. As is shown in [1], the parametric resonance, responsible for the effective energy transfer between different branches of excitations, determines the temporal evolution of the system, in particular, origin of the damping for the transverse breathing mode at zero temperature. The further consideration of the nonlinear evolution accompanying the parametric resonance [2] allows to explain the nontrivial picture of the damping revealed in the remarkable work of the Paris group [3]. The parametric amplification of the Bogoliubov modes due to periodic modulation of an optical 1D lattice is considered in [4]. The interesting picture of the density distribution in low-dimensional [5] and toroidal [6] Bose-condensed gases under conditions of the parametric excitation has been revealed at the numerical solution of the Gross-Pitaevskii equation.

A permanent modulation of the transverse trap frequency $\omega_{\perp}$ brings about the oscillations of the condensate density and, accordingly, sound velocity. Owing to the parametric resonance, this results in the generation of pairs of sound phonons with the opposite momenta and energies close to a half of the modulation frequency $\omega_{0} / 2$. In fact, phonon pairs are produced within the finite energy interval $\omega_{0} / 2 \pm E_{0}$. In what follows, this interval of parametric amplification with the width $2 E_{0}$ near $\omega_{0} / 2$ is identified as "the parametric interval". The parameter $E_{0}$ is connected with the modulation amplitude $\left|\delta \omega_{\perp} / \omega_{\perp}\right| \ll 1$ by the relation $E_{0} \sim \omega_{0} \cdot\left|\delta \omega_{\perp} / \omega_{\perp}\right|$. If $E_{0}$ exceeds the damping factor $\gamma$ for longitudinal phonons, their occupation numbers begin to increase exponentially. In this case the phonon-phonon interaction becomes significant. As a result, the evolution scenario changes drastically.

For the typical longitudinal size of traps $L$ a few phonon levels fall into the narrow energy interval $2 E_{0}$. The main effect of the phonon-phonon interaction, in addition to the damping, manifests itself in an effective renormalization of the levels. In the course of the self-consistent nonlinear evolution the position of levels changes continuously with changing the number of phonons. As is shown below, in the simplest case of one doubly-degenerate level in the parametric interval $2 E_{0}$, the evolution is over as the renormalized level reaches the left or right boundary of the interval. Then the growth of the phonon occupation numbers ceases. As a result, the steady state of phonons with the macroscopic occupation number in a single state and the common phase for all excitations appears.

In the case of several levels the picture is more sophisticated. It turns out that only one of the phonon levels finishes the evolution achieving the stationary state with the macroscopic number of phonons. As in the case of a single level, this level proves to be at the edge of the parametric interval.

The preliminary analysis of the problem for the case of a single level within the parametric interval has been represent in our work [8]. In the present paper we give a comprehensive consideration of the problem, including the general analysis of the evolution for arbitrary number of phonon levels within the parametric interval. There is considered the essential problem of indrawning outer levels located initially out of the energy interval of parametric amplification. Since in general case the evolution is over with the stationary occupation only a single level, the question about stability of this state is specially analysed. 


\section{PARAMETRIC GENERATION OF PHONON PAIRS}

1. Let us consider the Bose-condensed gas at $T=0$ in a trap of the cylindrical symmetry with $L \gg R$, where $R$ is the radius of the condensate. Neglecting the edge effects, we can write the general equation for the field operator $\hat{\Psi}(\mathbf{r}, z, t)$ of atoms in the form

$$
i \hbar \frac{\partial \hat{\Psi}}{\partial t}=\left[-\frac{\hbar^{2}}{2 m} \nabla_{\mathbf{r}}^{2}-\frac{\hbar^{2}}{2 m} \frac{\partial^{2}}{\partial z^{2}}+\frac{\omega_{\perp}^{2}(t) \mathbf{r}^{2}}{2}\right] \hat{\Psi}+U_{0} \hat{\Psi}^{+} \hat{\Psi} \hat{\Psi}
$$

where $U_{0}=4 \pi \hbar^{2} a / m, a$ being the $3 \mathrm{D}$ s-scattering length. Note, considering a dilute and cold Bose gas with dominant binary collisions we imply as usually that the radius of interaction region $r_{0} \ll a$, the gas parameter $n a^{3} \ll 1$, and the correlation length $\xi>n^{-1 / 3}$ ( $n$ is the atomic density). In this case, the replacement of two-body interaction with the effective contact potential is relevant. We assume that the trap transverse frequency depends on time as

$$
\omega_{\perp}(t)=\omega_{\perp}\left(1+\eta \sin \omega_{0} t\right), \quad \eta \ll 1
$$

According to Ref. [8], we introduce the spatial scaling parameter $b(t)$ and new variables $\vec{\rho}=\mathbf{r} / b(t), \tau(t)$. The field operator in terms of new variables can be written as

$$
\hat{\Psi}=\frac{\hat{\chi}(\vec{\rho}, z, \tau)}{b(t)} \cdot e^{i \Phi} ; \quad \Phi=\frac{m r^{2}}{2 \hbar} \cdot \frac{\dot{b}}{b}
$$

Substituting these expressions into Eq.(1), we obtain straightforwardly

$i \hbar \frac{\partial \hat{\chi}}{\partial \tau}=\left[-\frac{\hbar^{2}}{2 m} \nabla_{\rho}^{2}+\frac{m \omega_{\perp}^{2} \rho^{2}}{2}\right] \hat{\chi}+U_{0} \hat{\chi}^{+} \hat{\chi} \hat{\chi}-b^{2}(t) \frac{\hbar^{2}}{2 m} \frac{\partial^{2} \hat{\chi}}{\partial z^{2}}$,

provided that functions $b(t)$ and $\tau(t)$ satisfy the equations

$$
\frac{d^{2} b}{d t^{2}}+\omega_{\perp}^{2}(t) b=\frac{\omega_{\perp}^{2}}{b^{3}} ; \quad b^{2} \frac{d \tau}{d t}=1 .
$$

Treating the condensate wave function as independent of $z$, we find that Eq. (4) being expressed in variables $\rho, \tau$ describes the evolution at the time-independent frequency $\omega_{\perp}$. Combining the expression (2) with the condition $\omega_{0} \ll \omega_{\perp}$, we find the solution of Eq. (5) in the form

$$
b(t)=1+b_{1}(t), \quad b_{1}(t) \approx \frac{-\eta}{2} \cdot \sin \omega_{0} t .
$$

The field operator $\hat{\chi}$ can be represented in the usual form $\hat{\chi}=\left(\chi_{0}+\hat{\chi}^{\prime}\right) \exp (-i \mu \tau), \chi_{0}$ being the condensate wave function and $\mu$ is the initial chemical potential. In the absence of excitations at $T=0$ we can omit the last term in Eq. (4) and determine $\chi_{0}(\rho)$ from the equation

$$
\left[-\frac{\hbar^{2}}{2 m} \nabla_{\rho}^{2}+\frac{m \omega_{\perp}^{2} \rho^{2}}{2}-\mu\right] \chi_{0}+U_{0} \chi_{0}^{3}=0
$$

where $\mu$ is the chemical potential corresponding to the static cylindrical trap with $\omega_{\perp}=$ const. In the ThomasFermi limit when $n U_{0} \gg \hbar \omega_{\perp}$ the solution of this equation is reduced to known expression

$$
\chi_{0}^{2}=\frac{\mu}{U_{0}}\left(1-\frac{r^{2}}{R_{\perp}^{2} b^{2}(t)}\right) ; \quad R_{\perp}^{2}=\frac{2 \mu}{m \omega_{\perp}^{2}} .
$$

In the quasi-1D case when $n U_{0} \ll \hbar \omega_{\perp}$, we have

$$
\chi_{0}^{2}=\frac{n_{1}}{\pi l_{\perp}^{2}} \exp \left(-\frac{r^{2}}{l_{\perp}^{2} b^{2}(t)}\right) ; \quad l_{\perp}^{2}=\frac{\hbar}{m \omega_{\perp}} .
$$

In the both cases the modulation of the trap transverse frequency results in the oscillations of the condensate density.

2. Considering excited states, we first linearize Eq. (44) in $\hat{\chi}^{\prime}$. Then from Eq. (4) we have

$$
\begin{aligned}
& i \hbar \frac{\partial \hat{\chi}^{\prime}}{\partial \tau}=h_{0} \hat{\chi}^{\prime}+G\left(\hat{\chi}^{\prime}+\hat{\chi}^{\prime}\right)-b^{2}(t) \frac{\hbar^{2}}{2 m} \frac{\partial^{2} \hat{\chi}^{\prime}}{\partial z^{2}}, \\
& h_{0}=-\frac{\hbar^{2}}{2 m} \nabla_{\rho}^{2}+\frac{m \omega_{\perp}^{2} \rho^{2}}{2}+G-\mu ; \quad G=U_{0} \chi_{0}^{2}(\vec{\rho}) .
\end{aligned}
$$

Under condition $\omega_{0} \ll \omega_{\perp}$ only long-wave longitudinal phonons prove to be involved into the evolution of the system.

For these phonons the condition $k R \ll 1$ is fulfilled and the transverse distribution is close to $\chi_{0}$ (see, e.g., [9]). Comparison of Eqs. (7) and (8) allows us to simplify the latter equation omitting the first term in the right side. Using the second relation in (5) and going over to variable $t$, we arrive at the following equation

$$
i \hbar \frac{\partial \hat{\chi}^{\prime}}{\partial t}=\frac{G}{b^{2}(t)}\left(\hat{\chi}^{\prime}+\hat{\chi}^{\prime}\right)-\frac{\hbar^{2}}{2 m} \frac{\partial^{2} \hat{\chi}^{\prime}}{\partial z^{2}} .
$$

Involving the symmetry of the problem, we have for the operator $\hat{\chi}^{\prime}$ in second quantization

$$
\hat{\chi}^{\prime}=\sum_{k} \chi_{k} \hat{a}_{k} ; \quad \chi_{k}=\frac{e^{i k z}}{\sqrt{L}} \cdot \phi_{0}(\vec{\rho}),
$$

where $\hat{a}_{k}$ being the annihilation operator for atoms. At $\omega_{0} \ll \omega_{\perp}$ the function $\phi_{0}$ is actually close to $\chi_{0}$ for both the quasi-1D and Thomas-Fermi cases. In the expression (10) we keep the ground state alone for the transverse motion assuming that higher states are insignificant in the course of evolution. Substitution of the expression (10) into Eq.(9) gives after the standard averaging over the radial variables

$$
i \hbar \frac{d \hat{a}_{k}}{d t}=\left(\bar{G}+\frac{\hbar^{2} k^{2}}{2 m}\right) \hat{a}_{k}+\bar{G} \hat{a}_{-k}^{+}-\eta \bar{G} \sin \omega_{0} t\left(\hat{a}_{k}+\hat{a}_{-k}^{+}\right)
$$

$\bar{G}=\int d^{2} \rho \phi_{0}^{2}(\vec{\rho}) G(\vec{\rho})$. Let us rewrite this equation in terms of phonon operators using the usual transformation $\hat{a}_{k}=u_{k} \hat{b}_{k}+v_{k} \hat{b}_{-k}^{+}$. When the last term in Eq. (11) is omitted, a set of equations for $\hat{b}_{k}, \hat{b}_{-k}^{+}$determines the 
well-known Bogoliubov spectrum $\omega_{k}$ (with the replacement $\left.n U_{0} \rightarrow \bar{G}\right)$. Considering the nonstationary case, we introduce the substitution $\hat{b}_{k}=\hat{\tilde{b}}_{k} \exp \left(-i \omega_{0} t / 2\right)$ and take into account that long times $\omega_{0} t \gg 1$ are most interesting for the analysis. Then, within the resonance approximation, which implies that we discard the strongly oscillating terms with $\exp \left( \pm i \omega_{0} t / 2\right)$, the equations for $\hat{b}_{k}, \hat{b}_{-k}^{+}$ are reduced to the form

$$
i \frac{d \hat{\tilde{b}}_{k}}{d t}=\xi_{k} \hat{\tilde{b}}_{k}+i E_{0 k} \hat{\tilde{b}}_{-k}^{+} ; \quad-i \frac{d \hat{\tilde{b}}_{-k}^{+}}{d t}=\xi_{k} \hat{\tilde{b}}_{-k}^{+}-i E_{0 k} \hat{\tilde{b}}_{k},
$$

$E_{0 k}=(\eta \bar{G} / 2 \hbar)\left[\left(u_{k}+v_{k}\right) / u_{k}\right], \xi_{k}=\omega_{k}-\omega_{0} / 2$. For sound phonons $\left|\left(u_{k}+v_{k}\right) / u_{k}\right| \approx \hbar \omega_{k} / \mu$. Since $E_{0 k} \ll \omega_{0}$ and we are interested in the energy interval $\left|\omega_{k}-\omega_{0} / 2\right| \leq 2 E_{0}$, the frequency $\omega_{k}$ in the expression for $E_{0 k}$ can be replaced by $\omega_{0}$. In the other words, $E_{0 k}$ is replaced by its value at $k=\left|k_{0}\right|$, where $\left|k_{0}\right|$ is determined from the condition $\omega_{0}=2 \omega_{k_{0}}$. As a result, we have

$$
E_{0 k_{0}} \approx \eta \omega_{0}=\omega_{0} \frac{\left|\delta \omega_{\perp}\right|}{\omega_{\perp}} \equiv E_{0} .
$$

Substituting the transformation $\hat{\tilde{b}}_{k}=$ $c_{1 k} \exp \left(i \varepsilon_{0 k} t\right) \hat{\tilde{b}}_{k}^{\prime}+c_{2 k} \exp \left(-i \varepsilon_{0 k} t\right) \hat{\tilde{b}}_{-k}^{+^{\prime}}$ into (12), we find $\varepsilon_{0 k}=i \alpha_{0 k}$ with $\alpha_{0 k}=\left[\left|E_{0}\right|^{2}-\xi_{k}^{2}\right]^{1 / 2}$. This result demonstrates the appearance of the parametric resonance with an exponential growth of the phonon occupation numbers at $\left|E_{0}\right|>\left|\xi_{k}\right|$, which is induced by the modulation of the transverse trap frequency. Thus the parametric resonance occurs within the narrow range near $\omega_{0}$ with the width $2 E_{0}$.

\section{EVOLUTION EQUATIONS}

1. So far we have disregarded the phonon-phonon interaction. With an exponential growth of the phonon number the interaction begins to play an essential role. The weakness of the phonon-phonon interaction as itself (see below) allows us to take only three- and four-phonon processes into account. Assuming generation of sound phonons alone in the parametric interval $2 E_{0}$, we can use the expressions for $H^{(3)}$ and $H^{(4)}$ obtained within the hydrodynamic approximation, see [11]. Owing to smallness of the gas parameter, the dominant term in the Hamiltonian of three-phonon interactions has the form [11]

$$
H^{(3)}=\frac{m}{2} \int d^{3} r \hat{\mathbf{v}} \delta \hat{n} \hat{\mathbf{v}},
$$

Here $\hat{\mathbf{v}}, \delta \hat{n}$ are the operators of velocity and alternating part of the density, respectively. In fact, we consider the interaction of longitudinal sound phonons that effectively reduces $H^{(3)}$ to the one-dimensional problem.

Using the known expressions for operators $\hat{\mathbf{v}}, \delta \hat{n}$ [1], one can find the vertex $A_{3}$ for $H^{(3)}$ in (14).
Since each three-phonon vertex implies the momentum conservation law, at least one of three phonons lies beyond the parametric interval and, therefore, has zero occupation number at $T=0$. This makes possible to reduce the expression obtained in second order in $H^{(3)}$ to the effective Hamiltonian for the four-phonon interaction $(\Delta(k)$ is the Kroneker symbol)

$$
H_{e f f}=\frac{A}{2} \sum_{k_{1}, \ldots, k_{4}} \hat{b}_{k_{1}}^{+} \hat{b}_{k_{2}}^{+} \hat{b}_{k_{3}} \hat{b}_{k_{4}} \Delta\left(k_{1}+k_{2}-k_{3}-k_{4}\right) .
$$

Here all states lie within the interval of $2 E_{0}$. Using the expression for $A_{3}$ and taking into account that all wave vectors $\left|k_{i}\right| \sim k_{0}\left(E_{0} \ll \omega_{0}\right)$, we find (to the accuracy of numerical factor of the order of unity)

$$
|A| \approx \frac{\omega_{0}^{2}}{\mu N},
$$

where $N$ is the total number of particles.

The same four-phonon interaction can be obtained as a result of the standard canonical transformation of the Hamiltonian $H_{0}+H^{(3)}$ (see, for example, [12]).

The direct calculation shows that the ratio of the matrix element in $H^{(4)}$ from Ref. [11] to $|A|$ is proportional to $\left(n a^{3}\right)^{1 / 2} \ll 1$.

In addition, second order in $H^{(3)}$ contains the imaginary part related to real decay processes of phonons, which determine the phonon damping. The later we take into account phenomenologically introducing a decrement $\gamma$. After work [3] one can conclude that the parameter $\gamma$ is small at $T=0$ for the geometry under consideration. Therefore, it is rather easy to satisfy the conditions when $\alpha_{0 k}>\gamma$ and the parametric growth of the number of phonons remains.

2. As is follows from the above expressions, the Hamiltonian of interacting phonons near the parametric resonance have the form

$$
H=\sum_{k} \omega_{k} \hat{b}_{k}^{+} \hat{b}_{k}+\sum_{k}\left(E_{0} e^{-i \omega_{0} t} \hat{b}_{k}^{+} \hat{b}_{-k}^{+}+H . c .\right)+H_{e f f}
$$

Let us find $\left(d \hat{b}_{k} / d t\right)$ taking into account the phononphonon interaction. Using the substitution $\hat{b}_{k}=$ $\hat{b_{k}} \exp \left(-i \omega_{0} t / 2\right)$, we have

$$
\begin{aligned}
i \frac{d \hat{\tilde{b}}_{k}}{d t}= & \left(-i \gamma_{k}+\xi_{k}\right) \hat{\tilde{b}}_{k}+i E_{0} \hat{\tilde{b}}_{-k}^{+}+ \\
& +\sum_{k_{2} k_{3} k_{4}} A \tilde{\tilde{b}}_{k_{2}}^{+} \hat{\tilde{b}}_{k_{3}} \tilde{\tilde{b}}_{k_{4}} \Delta\left(k+k_{2}-k_{3}-k_{4}\right) .
\end{aligned}
$$

By means of the equations for $\hat{\tilde{b}}_{k}$ and $\hat{\tilde{b}}_{-k}^{+}$one can directly obtain the equations for the correlators $N_{k}=<\hat{\tilde{b}}_{k}^{+} \hat{\tilde{b}}_{k}>$ and $\hat{f}_{k}=<\hat{\tilde{b}}_{k} \hat{\tilde{b}}_{-k}>$. These equations contain the fourphonon correlators. Within the mean-field approximation we represent the four-phonon terms as products of 
the two-phonon correlators $N_{k}$ and $\hat{f}_{k}$. This implies that we keep the terms linear in $A$. In addition, we go over to the classical Bose field for phonons supposing that the number of parametrically excited phonons is large. As a result, we arrive at a set of nonlinear equations that describes the self-consistent evolution of interacting phonons within the parametric interval. These equations have the form

$$
\begin{aligned}
& \frac{d N_{k}}{d t}=-2 \gamma N_{k}+E_{0}\left(f_{k}+f_{k}^{*}\right)+i A\left(\mathcal{P}^{*} f_{k}-\mathcal{P} f_{k}^{*}\right) \\
& \frac{d f_{k}}{d t}=-2\left(\gamma+i \bar{\xi}_{k}\right) f_{k}+2 E_{0} N_{k}-2 i A \mathcal{P} N_{k}
\end{aligned}
$$

where the position of the renormalized level is determined by the expression

$$
\bar{\xi}_{k}=\xi_{k}+\mathcal{Q}+\bar{A} N_{k} ; \quad \bar{A}=3 A .
$$

Here $\xi_{k}$ is the level position at the initial time moment, $\mathcal{Q}=2 A \sum_{k^{\prime} \neq \pm k} N_{k^{\prime}} ; \mathcal{P}=\sum_{k^{\prime} \neq \pm k} f_{k^{\prime}}$. Hereafter the evident relations $\omega_{k}=\omega_{-k}, N_{k}=N_{-k}$ are used.

For the large occupation numbers $N_{k}$ to the accuracy of terms $\sim 1 / N_{k}$, the function $f_{k}(t)$ can be written in the form $f_{k}(t)=\left|f_{k}(t)\right| \exp \left[i \varphi_{k}(t)\right]=N_{k}(t) \exp \left[i \varphi_{k}(t)\right]$. As a result, we re-arrange (19) to a set of equations for $N_{k}$, $\varphi_{k}$

$$
\begin{gathered}
\frac{d N_{k}}{d t}=-2 \gamma N_{k}+2 E_{0} N_{k} \cos \varphi_{k}- \\
\quad-2 A N_{k} \sum_{k^{\prime} \neq \pm k} N_{k^{\prime}} \sin \left(\varphi_{k}-\varphi_{k^{\prime}}\right) \\
\frac{d \varphi_{k}}{d t}=-2 \bar{\xi}_{k}-2 E_{0} \sin \varphi_{k}-2 A \sum_{k^{\prime} \neq \pm k} N_{k^{\prime}} \cos \left(\varphi_{k}-\varphi_{k^{\prime}}\right),
\end{gathered}
$$

Hereafter we take into consideration that $\varphi_{k}=\varphi_{-k}$.

In what follows, we consider the case of finite longitudinal size $L$ assuming that a single or a few discrete two-fold degenerate levels lie within the energy interval of about $2 E_{0}$.

\section{SOLUTION FOR A SINGLE LEVEL}

1. In the case of a single level the equations (21) take the form

$$
\begin{aligned}
& \frac{d N_{k}}{d t}=-2 \gamma N_{k}+2 E_{0} N_{k} \cos \varphi_{k} \\
& \frac{d \varphi_{k}}{d t}=-2\left[\xi_{k}+\bar{A} N_{k}+E_{0} \sin \varphi_{k}\right] .
\end{aligned}
$$

This system has the following stationary solution

$$
N_{k}^{s}=\frac{\sqrt{E_{0}^{2}-\gamma^{2}} \mp \xi_{k}}{|\bar{A}|} ; \quad \sin \varphi_{k}^{s}=\mp \frac{\sqrt{E_{0}^{2}-\gamma^{2}}}{E_{0}} .
$$

The signs $\mp$ correspond to $\bar{A} \gtrless 0$, respectively. For definiteness, we suppose $E_{0}>0$. The result (23) has an interesting physical origin. In fact, the interaction $H_{\text {eff }}$ determines the effective renormalization of the phonon level, leading to $\delta \omega_{k}=\bar{A} N_{k}$ for the case concerned (see (20)). Accordingly, $\xi_{k} \rightarrow \bar{\xi}_{k}=\xi_{k}+\bar{A} N_{k}$. From Eqs. (23) it straightforwardly follows that $\bar{\xi}_{k}^{s}= \pm \sqrt{E_{0}^{2}-\gamma^{2}}$. So, at $N_{k}=N_{k}^{s}$ the renormalized level reaches the left or right edge of the parametric energy interval (within the accuracy of the shift due to $\gamma$ ). As a result, the parametric increase of the phonon occupation numbers stops and the phase acquires the constant value. The maximal value of the phonon number equals

$$
N_{k}^{s} \approx \frac{E_{0}}{|\bar{A}|} \gg 1 ; \quad \gamma, \xi_{k} \ll E_{0}
$$

The phase is $\varphi_{k}^{s} \approx \mp \pi / 2$ for $\bar{A} \gtrless 0$. The phase $\varphi_{k}$ corresponds to the phase correlation of phonon pairs with the opposite momenta. The appearance of the phase $\varphi_{k}$ and correlator $f_{k}$ is connected, first of all, with the creation of phonon pairs in the course of the evolution as a result of the parametric resonance. The evolution finishes in the stationary state of phonon pairs with zero total momentum and the common phase $\varphi_{k}^{s}$.

2. The character of the temporal evolution, tending asymptotically to the values (23), (24), depends essentially on the relations between the parameters in Eqs. (22). First, let $\gamma=0$. In this case, Eqs. (22) have the conserved integral of motion

$$
H_{0}=2 E_{0} N_{k} \sin \varphi_{k}+\bar{A} N_{k}^{2}+2 \xi_{k} N_{k}, \quad \frac{d H_{0}}{d t}=0 .
$$

The variables $N_{k}, \varphi_{k}$ are canonically conjugate, and the equation of motion (22) can be written in the Hamiltonian form

$$
\frac{d N_{k}}{d t}=\frac{\delta H_{0}}{\delta \varphi_{k}} ; \quad \frac{d \varphi_{k}}{d t}=-\frac{\delta H_{0}}{\delta N_{k}} .
$$

At the initial time moment, when the modulation of $\omega_{\perp}$ is switched on, we suppose $\bar{\xi}_{k}(0)=\xi_{k}+\bar{A} N_{k}(0)$ and $\bar{\alpha}_{0 k}=$ $\sqrt{E_{0}^{2}-\bar{\xi}_{k}(0)^{2}}$. At that, Eqs.(22) imply that $\cos \varphi_{k}(0)=$ $\bar{\alpha}_{0 k} / E_{0}$. Bearing in mind that $\sin \varphi_{k}(0)=-\bar{\xi}_{k}(0) / E_{0}$, from the second equation in (22) we obtain $\left(d \varphi_{k} / d t\right)(0)=$ 0 . As a consequence, the initial (and conserved) value of $H_{0}$ is equal to

$$
H_{0} \approx-\bar{A} N_{k}^{2}(0) \ll E_{0}
$$

For an arbitrary time, Eq. (25) can be rewritten as $2 E_{0} \sin \varphi_{k}(t)=-\left[2 \xi_{k}+\bar{A} N_{k}(t)\right]-H_{0} / N_{k}(t)$. Substituting this relation into equations (22), one can find

$$
\frac{d N_{k}}{d t}= \pm 2 N_{k} \alpha ; \quad \frac{d \varphi_{k}}{d t}=-\bar{A} N_{k}-\frac{H_{0}}{N_{k}},
$$

$\alpha=\left[E_{0}^{2}-\left(\bar{A} N_{k} / 2+\xi_{k}-H_{0} / 2 N_{k}\right)^{2}\right]^{1 / 2}$. The signs \pm correspond to the regions with $\left|\varphi_{k}\right| \leq \pi / 2$ and $\left|\varphi_{k}\right|>$ 
$\pi / 2$, respectively. For the region with $\left|\varphi_{k}\right| \leq \pi / 2$, the solution can be found straightforwardly

$$
2 t \approx \int_{N_{k}(0)}^{N_{k}(t)} \frac{d x}{x\left[E_{0}^{2}-\left(\bar{A} x / 2+\xi_{k}\right)^{2}\right]^{1 / 2}},
$$

Here we omitted the small term with $\left|H_{0}\right| \ll E_{0}$. The upper limit of the integral is equal to the value $N_{k}^{m} \approx$ $2\left(E_{0} \mp \xi_{k}\right) /|\bar{A}|$ at which the denominator vanishes. The divergence at $N_{k} \rightarrow 0$ is a typical manifestation of the parametric resonance which requires a nonzero initial field amplitude. This can be achieved by taking zeropoint oscillations into account [1]. Owing to these oscillations, we can put $N_{k}(0) \sim 1$. The time necessary for the system to achieve the maximal value $N_{k}^{m}$ is equal to

$$
t_{m} \approx \frac{1}{2 \alpha_{0 k}} \ln \left[\frac{4 E_{0}}{\bar{A}} \cdot\left(1-\frac{\xi_{k}^{2}}{E_{0}^{2}}\right)\right]
$$

We see that the argument of logarithm is much greater than unity at $\xi_{k}<E_{0}$ and $E_{0} \gg \bar{A}$. This implies that $t_{m} \gg 1 / 2 \alpha_{0 k}$ where $1 / 2 \alpha_{0 k}$ is the characteristic time of the parametric resonance. At $t=t_{m}$ we have $\left|\varphi_{k}\right|=\pi / 2$. As it follows from the second equation in (27), at $t>t_{m}$ the phase proves to be in the region with $\left|\varphi_{k}\right|>\pi / 2$. As a result, in the first equation the sign becomes negative and the phonon number reduces. This is the start of an oscillating behaviour. The numerical simulation shows that the joint solution of Eqs. (27) has the form of anharmonic oscillations of both the phonon number and the phase around their stationary values. At $\xi_{k}=0$ the exact solution for $N_{k}(t)$ can be written in terms of dn Jacobian elliptic function as

$$
N_{k}(t)=N_{k}^{m} d n\left[2 E_{0}\left(t-t_{m}\right) ; k\right], \quad N_{k}^{m}=\frac{2 E_{0}}{|\bar{A}|} .
$$

Where $d n(u ; k)=d n(-u ; k), d n(0 ; k)=1 ; k$ is the elliptic modulus [13]. For the given initial conditions we have $\sqrt{1-k^{2}}=\left|H_{0} \bar{A}\right| / 4 E_{0}^{2} \ll 1$.

3. The character of evolution changes drastically at $\gamma \neq 0$. Now in all cases the system asymptotically approaches the stationary state. At any set of initial parameters the solution of Eq. (22) leads to the values (24) for these stationary states. The damping of the oscillations has the decrement, which is close to $\gamma$, when $\alpha_{0} \gg \gamma$ and $t>t_{m}$. As $\gamma$ increases, the arrival time to the stationary state decreases as $1 / \gamma$. When $\gamma$ is relatively close to $\alpha_{0 k}$, the oscillations disappear. In this case the time necessary to reach the stationary state is allied to the value (29), if $\alpha_{0 k}$ is replaced by $\alpha_{1}=\alpha_{0 k}-\gamma$.

The direct numerical simulation of the system (22) demonstrates the picture described. As an example, in Fig.1 we report the results for the phonon number $N_{k}$ as a function of the dimensionless parameter $s=2 \gamma t$

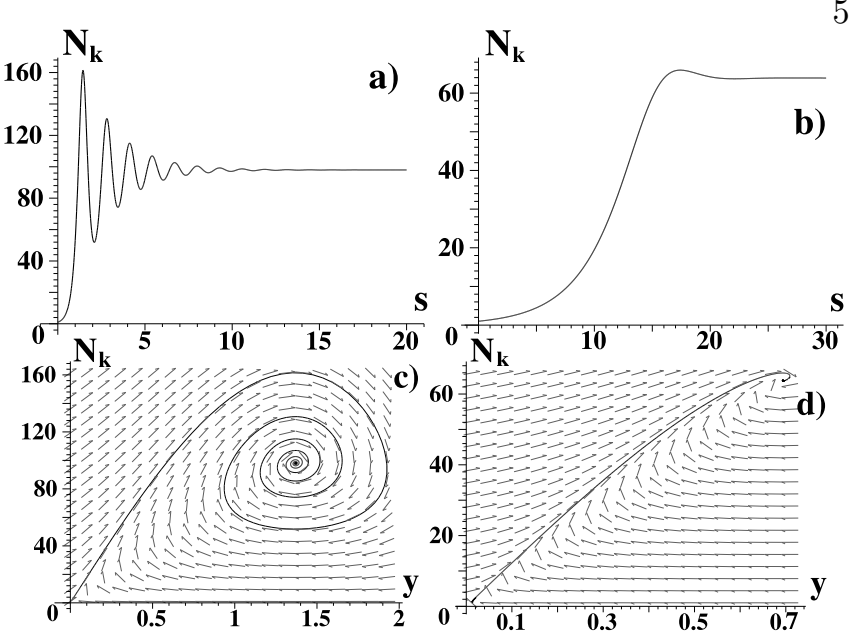

FIG. 1: Phonon numbers $N_{k}(s)$ versus $s=2 \gamma t(\mathrm{a}, \mathrm{b})$ and versus $y(t) \equiv-\varphi(t)(\mathrm{c}, \mathrm{d})$ for $\left(E_{0} / \gamma\right)=5,1.3$.

for $E_{0} / \gamma=5$ (a) and $E_{0} / \gamma=1.3$ (b). Figs. 2 c), d) demostrate the phase portraits in the plane $\left[N_{k}, \varphi_{k}\right]$ for these cases. (The parameters $\left(E_{0} / \bar{A}\right)=10^{2}$ and $\xi=0$ are used for the calculations.) In the both cases $N_{k}(t), \varphi_{k}(t)$ are assymptotically approaching to the values (23).

Thus at any finite values of $\gamma$ the self-consistent evolution of interacting phonons experienced the parametric amplification results in the formation of the stationary state with the macroscopic number (of the order of $E_{0} / \bar{A}$ ) of phonons in a single state.

It should be noted that the character of the temporal evolution is sign-independent of $\bar{A}$.

It is interesting to verify the stability of the solution obtained. It seems that the damping of fluctuations of the phonon number $\delta N=N_{k}-N_{k}^{s}$ may have a different character within and beyond the parametric interval. Really, at $\delta N<0$ the phonon number reverts to the stationary state owing to parametric pumping. While at $\delta N>0$, the absorption alone, determined by $\gamma$, returns the phonon system to this state.

Linearizing Eqs. (22) in $\delta N$ and $\delta \varphi=\varphi_{k}-\varphi_{k}^{s}$ with the initial conditions $\delta N(0)=N_{k}(0)-N_{k}^{s}$ and $\delta \varphi(0)=0$, we obtain

$$
\begin{aligned}
& \frac{d \delta N}{d t}=-\left[2 E_{0} N_{k}^{s} \sin \varphi_{k}^{s}\right] \delta \varphi \\
& \frac{d \delta \varphi}{d t}=-2 \bar{A} \delta N-\left[2 E_{0} \cos \varphi_{k}^{s}\right] \delta \varphi
\end{aligned}
$$

where $N_{k}^{s}$ and $\sin \varphi_{k}^{s}$ are determined by Eqs. (23). Taking into account that $\bar{A} \sin \varphi_{k}^{s}<0$ for the both signs of $\bar{A}$, we find the solution of Eqs. (32) in the form

$$
\begin{aligned}
& \delta N(t)=\delta N(0) e^{-\gamma t}\left(\cos \omega t-\frac{\gamma}{\omega} \sin \omega t\right) \\
& \delta \varphi(t)=\frac{-2 \delta N(0) e^{-\gamma t}|\bar{A}|}{\omega} \cdot \sin \omega t \\
& \omega^{2}=4\left(E_{0}^{2}-\gamma^{2}\right)^{1 / 2} \cdot\left[\left(E_{0}^{2}-\gamma^{2}\right)^{1 / 2}-\xi_{k}\right]-\gamma^{2} .
\end{aligned}
$$


This result demonstrates the stability of the stationary state against fluctuations of the phonon density. The character of relaxation to the stationary state turns out sign-independent of $\delta N(0)$.

The quantitave character of the solution with the damping fluctuations remains for the case $\delta \varphi(0) \neq 0$.

\section{SOLUTION FOR THE CASE OF SEVERAL LEVELS}

1. Let us consider the case when several discrete levels lie within the parametric interval. First of all, we analyze in detail the evolution to the stationary state for two levels at $\gamma \neq 0$. In the case of two levels, taking into account double degenaracy of each level in Eq. (21) and introducing notations $N_{k_{i}}=N_{-k_{i}}=N_{i} ; \varphi_{k_{i}}=\varphi_{-k_{i}}=$ $\varphi_{i} ; i=1,2$, we obtain

$$
\begin{aligned}
& \frac{d N_{1}}{d t}=-2 \gamma N_{1}+2 E_{0} N_{1} \cos \varphi_{1}- 4 A N_{1} N_{2} \sin \left(\varphi_{1}-\varphi_{2}\right) ; \\
& \frac{d \varphi_{1}}{d t}=-2 \xi_{1}-2 E_{0} \sin \varphi_{1}-6 A N_{1}-8 A N_{2}--4 A N_{2} \cos \left(\varphi_{1}-\varphi_{2}\right) ; \\
& \frac{d N_{2}}{d t}=-2 \gamma N_{2}+2 E_{0} N_{2} \cos \varphi_{2}+4 A N_{1} N_{2} \sin \left(\varphi_{1}-\varphi_{2}\right) ; \\
& \frac{d \varphi_{2}}{d t}=-2 \xi_{2}-2 E_{0} \sin \varphi_{2}-6 A N_{2}-8 A N_{1}- \\
&-4 A N_{1} \cos \left(\varphi_{1}-\varphi_{2}\right) ;
\end{aligned}
$$

Here the position of the renormalized level is determined by the expression (see (201))

$$
\bar{\xi}_{1}=\xi_{1}+3 A N_{1}+4 A N_{2} ; \quad \xi_{1}=\omega_{1}-\frac{\omega_{0}}{2} ;
$$

and, correspondently, for $\bar{\xi}_{2}$ with the replacement $1 \rightleftarrows 2$. The initial conditions are similar to those in the case of a single level, namely, $N_{1,2}(0) \sim 1 \quad \sin \varphi_{i}(0) \approx-\xi_{i} / E_{0}$.

The solution of Eqs. (33) demonstrates an interesting asymptotic picture. For any set of the parameters in Eqs. (33), the number of phonons corresponding to one of two levels vanishes. Owing to renormalization determined by Eq. (34), another level proves to be at the edge of the parametric interval with the number of phonons equal to $N_{i}^{s}$ from Eq.(23).

The disappearance of phonons at one of the levels is due to the following. This level, as a result of renormalization, proves to be either beyond the parametric region in which pumping is absent, or within the interval beside its edge where the damping exceeds the resonance pumping. In the both cases the growth of the phonon occupation numbers is suppressed by the damping.

In most cases we have the characteristic picture represented in Fig.2 when the level 1 closer to the left edge of

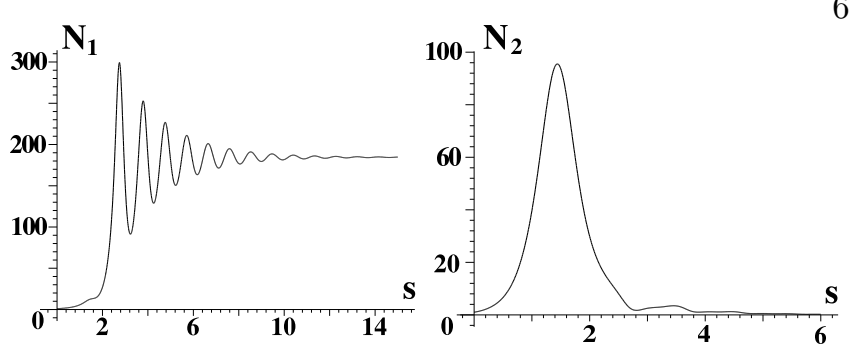

FIG. 2: Phonon numbers $N_{1,2}$ versus $s=2 \gamma t$ for $\xi_{1} / E_{0}=$ $-0.87, \xi_{2} / E_{0}=0.3$ and $E_{0} / \gamma=5$.

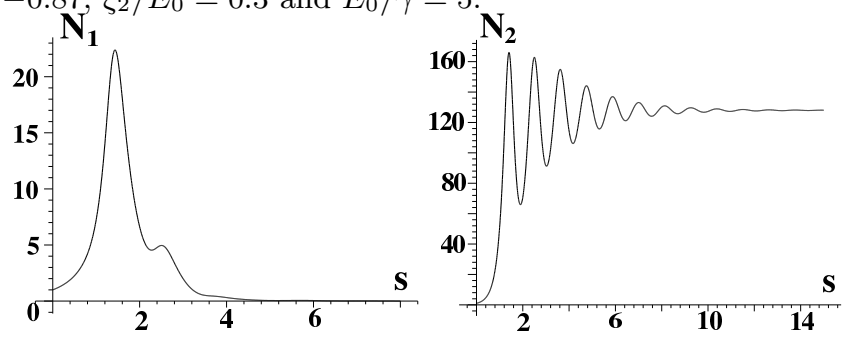

FIG. 3: Phonon numbers $N_{1,2}$ versus $s=2 \gamma t$ for $\left(E_{0} / \gamma\right)=5$ and $\left(\xi_{1} / E_{0}\right)=-0.87,\left(\xi_{2} / E_{0}\right)=-0.3$.

the interval at the initial time moment ("the left level") reaches the stationary state $\left(E_{0}, \bar{A}>0\right)$.

It is interesting that, owing to renormalization, the level 2 closer to the right edge of the parametric interval ("the right level") asymptotically proves to be far from the edge, $\bar{\xi}_{2} / E_{0}=2.75$.

Figure 3 demonstrates another characteristic situation. Now the right level $\xi_{2}$ comes to the stationary state. The renormalization, due to $N_{2}$, shifts the level $\xi_{1}$ to the right edge of the parametric interval, $\bar{\xi}_{1} / E_{0}=0.84$, where the subsequent evolution of the moderate values of $N_{1}$ is suppressed by $\gamma$.

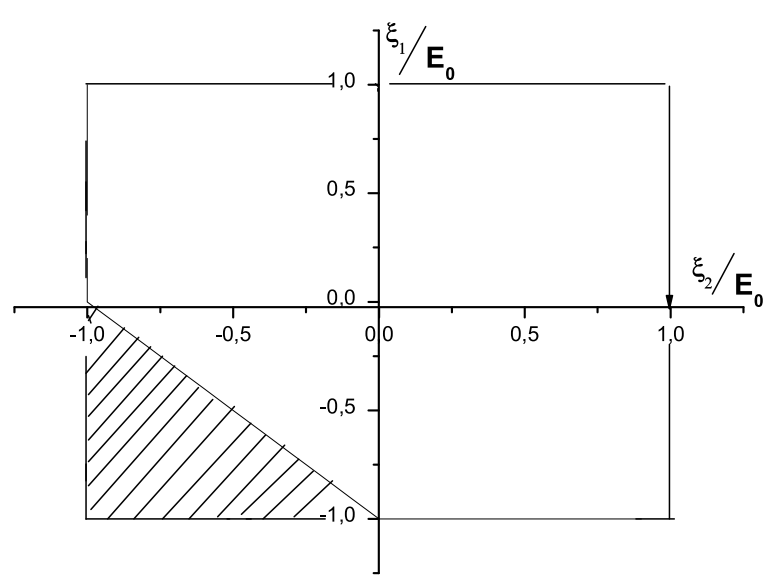

FIG. 4: In the shaded region the right level comes to the stationary state. In the rest part of the plane $\left[\xi_{1} ; \xi_{2}\right]$ the left level arrives at this $\operatorname{state}\left(E_{0} / \gamma=5\right)$

Figure 4 displays two regions in the plane $\left[\xi_{1}, \xi_{2}\right]$ for the fixed value $\left(E_{0} / \gamma\right)=5$. For the positions $\xi_{1}, \xi_{2}$ from the shaded region, the right level $\xi_{2}$ arrives at the stationary state. For the other positions, the left level $\xi_{1}$ 
reaches this state.

The essential remarks should be added. In principle, owing to the renormalization, an outer level with $\left|\xi_{1}\right| / E_{0}>1\left(\xi_{1}<0\right)$ can be indrawn into the parametric interval. It is impossible for any ratio $E_{0} / \gamma$, if $\xi_{2}<0$. This is already clear from Fig.4 (with decreasing $E_{0} / \gamma$ the shaded region only enlarges).

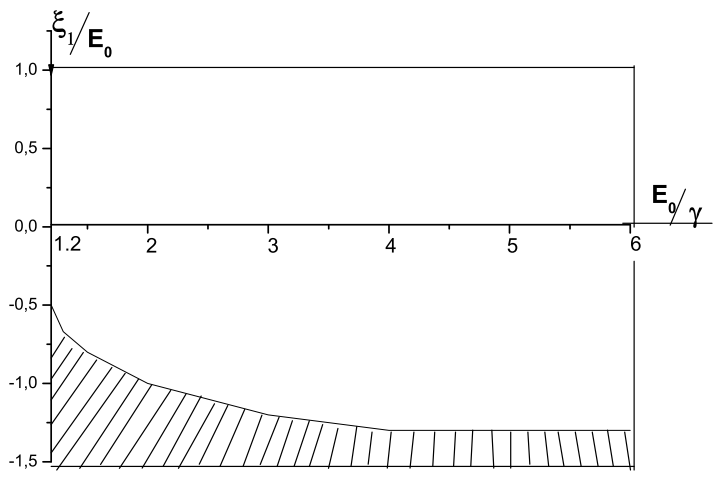

FIG. 5: The shaded region covers the level positions at the fixed ratio $\xi_{2} / E_{0}=0.4$, including the levels with $\xi_{1} / E_{0}<$ -1 , which are damped in the course of the evolution. The stationary state is approached by the state with $\xi_{2}$.

However, it is possible, when $\xi_{2}>0$. Moreover, the indrawn level can assymptotically approach the edge of the parametrical interval forming the stationary state. For any $\xi_{2}>0$ there is the limited interval $\delta_{c}\left(\xi_{2}\right)=$ $\left(\left|\xi_{1 c}\right|-E_{0}\right) / E_{0}$, when this event is not realized. At $\delta<\delta_{c}$ the indrawn level ousts the level $\xi_{2}$ and reaches the stationary position as long as the ratio $E_{0} / \gamma$ exceeds some critical value. The interval $\delta_{c}$ increases with decreasing $\xi_{2}$. The direct numerical calculation gives $\left(\delta_{c}\right)_{\max } \approx 0.6$.

As an illustration, in Fig.5 we plot the plane $\left[\xi_{1} / E_{0} ; E_{0} / \gamma\right]$ and distinguish two regions for the fixed value $\xi_{2} / E_{0}=0.4$. In the shaded region the positions of left levels, including the ones with $\xi_{1} / E_{0}<-1$, which are damped in the course of the evolution, are collected. In this case the level with $\xi_{2}$ comes to the stationary state. The rest part of the plane is occupied by the level positions (including again indrawn levels at $\xi_{1} / E_{0}<-1$ ) for which the left level ends the the evolution in the stationary state with the macroscopic number of phonons (here we imply that $\xi_{1}<\xi_{2}$ ).

Actually, all features discussed here are represented in Fig.5.

3. All qualitative features revealed for two levels are reproduced for the case of many levels within the parametric interval. First of all, in the course of the evolution only one level proves to be in the stationary state.The population of other levels is going to zero in all cases. The picture of the evolution as itself can demonstrate different patterns. In this aspect already the case of three

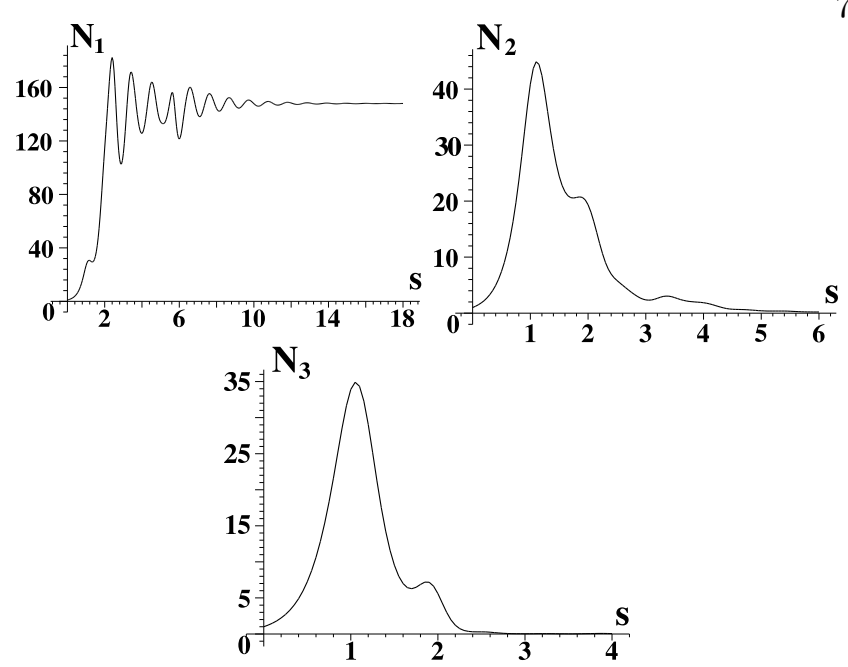

FIG. 6: Phonon numbers $N_{1,2,3}$ versus $s=2 \gamma t$ for $\left(\xi_{1} / E_{0}\right)=$ $-0.5,\left(\xi_{2} / E_{0}\right)=-0.1,\left(\xi_{3} / E_{0}\right)=0.1$ and $\left(E_{0} / \gamma\right)=5$.

levels shown as an illustration in Figs. 6-8 is quite instructive. Really, in Fig.6 the leftmost level 1 is eventually found at the stationary state, while two other levels are pushed out of the parametric interval, $\bar{\xi}_{2} / E_{0}=1.83$, $\bar{\xi}_{3} / E_{0}=2.03$.

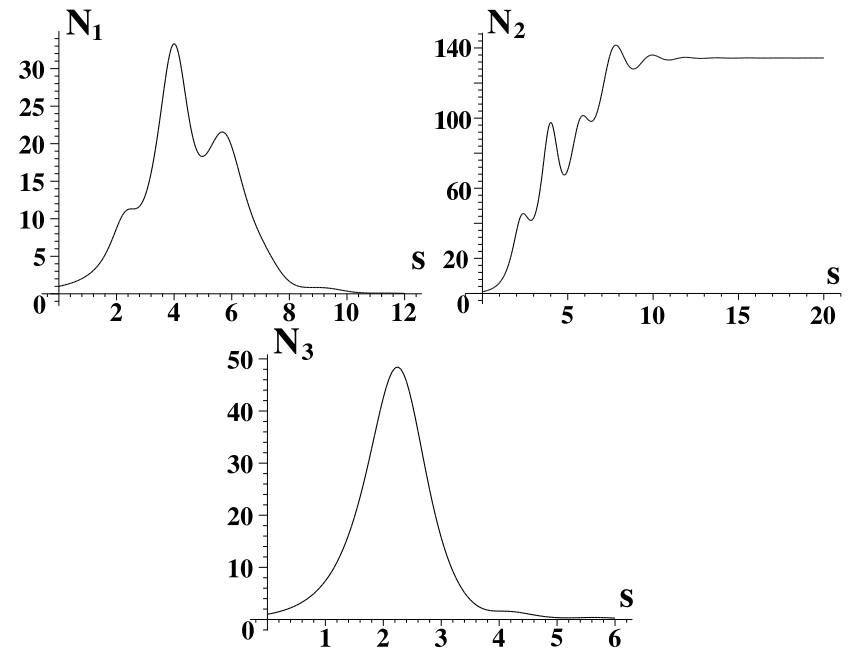

FIG. 7: Phonon numbers $N_{1,2,3}$ versus $s=2 \gamma t$ for $\left(E_{0} / \gamma\right)=$ $3,\left(\xi_{1} / E_{0}\right)=-0.8,\left(\xi_{2} / E_{0}\right)=-0.4,\left(\xi_{3} / E_{0}\right)=0.1$.

In Fig. 7 the intermediate level 2 comes to the stationary state. Now only the rightmost level is ousted from the parametric interval $\left(\bar{\xi}_{3} / E_{0}=1.89\right)$. The leftmost level 1 ends its evolution with zero population at $\bar{\xi}_{1} / E_{0}=0.9$

At last, Figure 8 displays the interesting situation when two levels lie beyond the parametric interval, although they are sufficiently close to the edge. In this case the both levels are indrawn into the interval. The leftmost level arrives at the stationary state.

It should be emphasized that in all cases the stationary state arising in the course of the evolution has the number of phonons and the phase equal to the values determined 


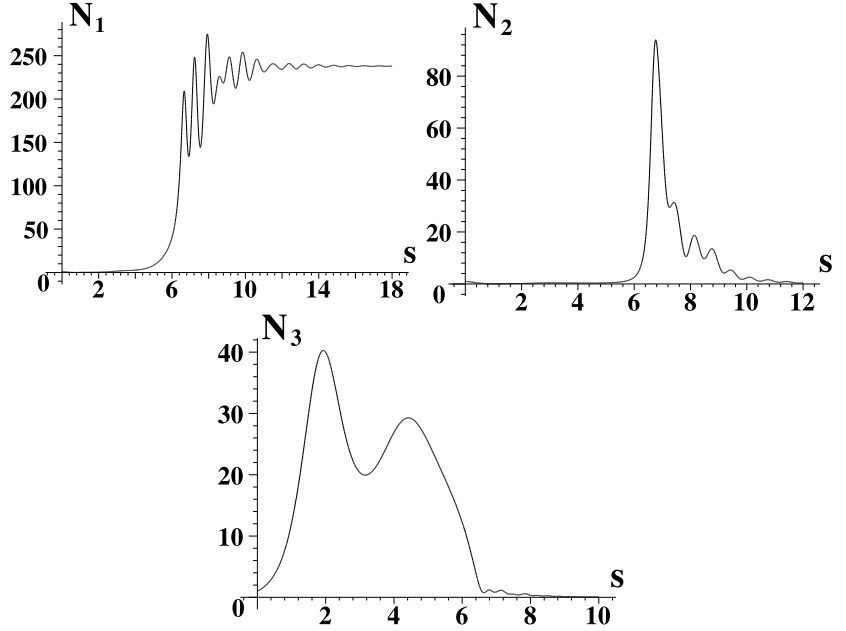

FIG. 8: Phonon numbers $N_{1,2,3}$ versus $s=2 \gamma t$ for $\left(E_{0} / \gamma\right)=$ $5,\left(\xi_{1} / E_{0}\right)=-1.4,\left(\xi_{2} / E_{0}\right)=-1.2,\left(\xi_{3} / E_{0}\right)=0.7$.

by the expressions (23).

To conclude, we note that the character of the evolution described above holds and for $A<0$. In this case the levels move to the left edge of the parametric interval in the course of the evolution.

\section{PERIODICAL STUCTURE AND SOME ESTIMATIONS}

1. The self-consistent temporal evolution of interacting phonons near the parametric resonance results in the formation of the stationary state with the macroscopic phonon number of about $E_{0} / \bar{A}$ in a single quantum state with the common phase for all excitations. The stationary state has one of two energies corresponding to the right or left edge of the parametric interval. In fact, the stationary state is formed by the phonon pairs which are a coherent superposition of two states with the wave vectors $k_{s}$ and $-k_{s}$ where $k_{s}=\omega_{s} / c_{0}$ and $\omega_{s}=\omega_{0} / 2 \pm E_{0}$.

This state generates the stationary space modulation of the atomic condensate. This is easy to reveal determining the atomic density as $\delta n=<\hat{\chi}^{\prime}+(\rho, z) \hat{\chi}^{\prime}(\rho, z)>$, where $\hat{\chi}^{\prime}$ has the form (10). Rewriting $\hat{\chi}^{\prime}$ in terms of phonon operators and integrating over $d^{2} \rho$, we arrive at the expression for the modulation of the $1 \mathrm{D}$ atomic density

$$
\frac{\delta n(z)}{n^{(1)}}=\frac{2 N_{k}^{s}}{N}\left(u_{k_{s}}^{2}+v_{k_{s}}^{2}\right) \cos 2 k_{s} z \approx \frac{2 \mu}{\varepsilon_{s}} \cdot \frac{N_{k}^{s}}{N} \cdot \cos 2 k_{s} z,
$$

where $n^{(1)}=N / L$. We suppose that $|\delta n| / n^{(1)} \ll 1$. The phase $\varphi_{k}$ does not enter into this expression owing to the accepted relation $\varphi_{k}=\varphi_{-k}$.

It should be emphasized that the static periodical structure (35) is generated by the pair correlations between phonons and has the amplitude proportional to the number of quasiparticles in the stationary phonon state with the macroscopic population.
2. Let us make some quantitative estimates. For definiteness, we consider the quasi 1D situation. As is known, in this case the chemical potential is equal to $\mu=\omega_{\perp} s$ where $s=a^{(3)} n^{(1)}, a^{(3)}$ is the 3D scattering length (see 14]). The quasi 1D situation takes place under condition $s<1$. Determining the stationary magnitude of the number of phonons, we use expressions (13), (16) and (23) supposing $E_{0} \gg \gamma, \xi_{k}$. As a result, we find

$$
N_{k}^{s} \approx \frac{E_{0}}{|\bar{A}|} \approx N\left(\frac{\omega_{\perp}}{\omega_{0}}\right) \eta s ; \quad \eta=\frac{\left|\delta \omega_{\perp}\right|}{\omega_{\perp}} .
$$

Using this value and definition of the chemical potential, we obtain the following expression for the amplitude of the periodic term in the longitudinal condensate density

$$
\frac{|\delta n|}{n^{(1)}} \approx\left(\frac{\omega_{\perp}}{\omega_{0}}\right)^{2} s^{2} \eta
$$

To estimate the number of the phonon levels $N_{0}$ within the parametric interval $2 E_{0}$, we take into account that the distance between the nearest levels is equal to $\delta \omega_{\|}=$ $2 \pi c_{0} / L$, where $c_{0}=\sqrt{\mu / 2 m}$ is the averaged value of the longitudinal sound velocity. Then

$$
N_{0}=\frac{2 E_{0}}{\delta \omega_{\|}}=\frac{\sqrt{2}}{\pi} \frac{\eta}{\sqrt{s}} \cdot \frac{\omega_{0}}{\omega_{\perp}} \cdot \frac{L}{l_{\perp}},
$$

where $l_{\perp}=\sqrt{1 / m \omega_{\perp}}$. Let us consider a set of parameters acceptable for experiment

$$
\begin{gathered}
N=10^{4} ; \quad L=2 \cdot 10^{-2} \mathrm{~cm} ; \quad \omega_{\perp}=10^{4} \mathrm{sec}^{-1} ; \\
\frac{\omega_{0}}{\omega_{\perp}}=0.2 ; \quad \eta=10^{-2} .
\end{gathered}
$$

For rubidium atomic gas we arrive at the following estimates

$$
s=0.25 ; \quad N_{k}^{s} \approx 10^{2} ; \quad \frac{|\delta n|}{n^{(1)}} \approx 10^{-1} ; \quad N_{0} \sim 1 .
$$

We see that all conditions assumed before are satisfied.

Thus we can conclude that the state with the macroscopic number of phonons can be realized for a realistic set of parameters.

\section{CONCLUSION}

The analysis presented in the paper has revealed the nontrivial picture of the temporal evolution of phonons created in a Bose-condensed atomic gas as a result of the parametric resonance. The phonon-phonon interaction is shown to play a decisive role here. The interaction leads to the renormalization of phonon levels which increases with growing the number of phonons. It turns out that in all cases the evolution stops, when one of phonon levels within the parametric interval approaches the edge of this interval (phonon losses are compensated by the 
permanent pumping). The macroscopic population of a single level is characteristic for this event. At the same time the population of all other levels is asymptotically going to zero. Actually, the stationary level is filled by pairs of correlated phonons with the opposite momenta $k_{s},-k_{s}$ and the common phase. Thus the self-consistent nonlinear evolution under consideration has completed with the formation of the condensate composed of two parts. One part is still in the initial state. The second part is made up of the macroscopic number of phonons and gives rise to the modulation in the atomic density.

It should be noted that, for the finite but sufficiently low temperatures $T \lesssim \hbar \omega_{0}$, when the initial number of phonons with $\omega_{k} \sim \omega_{0}$ is limited, the results obtained remain valid.

This work is supported by the Russian Foundation for Basic Research (Grant No.07-02-00067a) and by the Grant for Russian Science Schools (No.NS-6869.2006.2).

*manakova@kurm.polyn.kiae.su
[1] Yu.Kagan and L.A.Maksimov, Phys.Rev.A, 64, 053610 (2001).

[2] Yu.Kagan and L.A.Maksimov, Phys.Lett.A, 317, 477 (2003).

[3] F.Chevy, V.Bretin, P.Rosenbusch, K.W.Madison, and J.Dalibard, Phys.Rev.Lett., 88, 250402 (2002).

[4] M.Kraemmer, C.Tozzo, and F.Dalfovo, Phys.Rev.A, 71, 023613 (2005).

[5] K. Staliunas, S.Longhi, and G.J.de Valcarcel, Phys.Rev.A, 70, 011601(R) (2004).

[6] M.Modugno, C.Tozzo, and F.Dalfovo, cond-mat/0605183.

[7] Yu.Kagan and L.A.Manakova, cond-mat/0609159 Phys.Lett.A, 361, 401 (2007).

[8] Yu.Kagan, E.L.Surkov and G.V.Shlyapnikov, Phys.Rev.A, 54, R1753 (1996).

[9] E.Zaremba, Phys.Rev.A, 57, 518 (1998).

[10] S.Stringari, Phys.Rev.A, 58, 2385 (1998).

[11] E.M.Lifshitz and L.P.Pitaevskii, Statistical Physics (Pergamon, Oxford, 1980), Part 2.

[12] M.Wagner, Unitary Transformation in Solid State Physics, North-Holland, Amsterdam, 1986

[13] M. Abromovitz and I.A.Stegun, Handbook of Mathematical Functions, Dover, New York, 1970.

[14] M.Olshanii, Phys.Rev.Lett., 81, 938 (1998). 